\title{
Survey on War Soldiers Health Care Monitoring System Using Machine Learning and IOT
}

\author{
Mr. Abhilash ${ }^{1}$, Ms. Sathya Shree K P ${ }^{2}$, Ms. Subiya Seher ${ }^{3}$, Ms. Thanusha $S^{4}$ \\ Assistant Professor, Dept. of Information Science \& Engg., Atria Institute of Technology, Bangalore, India ${ }^{1}$ \\ Student, Dept. of Information Science \& Engg., Atria Institute of Technology, Bangalore, India ${ }^{2,3,4}$
}

\begin{abstract}
The army soldiers play a vital role to secure the nation. There are many concerns regarding the safety of soldiers. The objective of this project is to develop a security device which gives an ability to track the location and monitor health of the soldiers in real time who become lost and get injured in the battle field. This system will enable army control unit to track the location and monitor health of soldiers using GPS module and wireless body area sensor networks. The data coming from sensors and GPS receiver will be transmitted to the fellow soldiers wherein they are directed so that they can help and rescue the injured one and get him/her to the nearest medical camp using the same GPS module system.
\end{abstract}

Keywords: Global Positioning System, Zigbee, Wireless Sensor Network

\section{INTRODUCTION}

The Internet of things is the inter-networking of physical devices, vehicles, buildings and other items - embedded with electronics, software, sensors, actuators, and network connectivity that enable these objects to collect and exchange data. Machine Learning is an idea to learn from examples and experience, without being explicitly programmed. Instead of writing code, you feed data to the generic algorithm, and it builds logic based on the data given.

\section{RELATED SURVEY}

A Soldier Health Monitoring System for Military Applications can be implemented with recent advances in technology, various wearable sensors have been developed for the monitoring of human physiological parameters. A Body Sensor Network (BSN) consisting sensors such as biomedical sensors and physiological sensors placed on, near or within a human body can be used for real - time health monitoring. In this paper, there is description of an on-going effort to develop a system consisting of interconnected BSNs for real-time health monitoring of soldiers. We describe the prototype of the system and present a blast localization application.

In recent years, there have been tremendous advances in the development of Wireless Sensor Networks (WSNs) technologies. A WSN usually comprises a large number of low-cost, low-power and tiny sensor nodes, each consisting of sensors, microcontroller, memory, and radio transceiver.

There are many applications of WSNs, including battlefield surveillance, environmental monitoring, health monitoring, smart spaces, industrial diagnostics, etc. As a member of the WSN family, a Body Sensor Network (BSN) is composed of various physiological and biomedical sensor nodes that are placed on, near or within a human body to monitor parameters such as core temperature, heart rate, EEG, ECG, etc

This system is intended to be used for real-time monitoring and reporting of soldier's health information including physiological and cognitive status. later, we describe a preliminary prototype implementation of the BSN component of the system, including its hardware platform, system architecture and network protocol stack. We also present an application of the system for blast source localization.

For the blast impact assessment, we propose a blast source localization algorithm. The idea of the algorithm is to make use of the acceleration experienced by each soldier due to the blast to estimate the location of the blast.

The paper also proposes further integration with existing equipment in use. Effective interfacing has been achieved and results suggest proper intimation of pulse rate and location as soon as pulse values drop below the pre-decided threshold or on receiver demand. Robustness of the entire device may be enforced to help it beat all the environmental and climatic challenges at the war fronts and national borders. The rigorous analysis may be performed for integrating 
the proposed system with existing paraphernalia (used by army personnel) which may yield in budget-friendly and reliable implementation. This study may be extended to ensure the healthcare and well being of the citizens of the world as well. The paper also proposes further integration with existing equipment in use. Effective interfacing has been achieved and results suggest proper intimation of pulse rate and location as soon as pulse values drop below the pre-decided threshold or on receiver demand. Robustness of the entire device may be enforced to help it beat all the environmental and climatic challenges at the war fronts and national borders. The rigorous analysis may be performed for integrating the proposed system with existing paraphernalia (used by army personnel) which may yield in budgetfriendly and reliable implementation. This study may be extended to ensure the healthcare and well being of the citizens of the world as well. The paper also proposes further integration with existing equipment in use. Effective interfacing has been achieved and results suggest proper intimation of pulse rate and location as soon as pulse values drop below the pre-decided threshold or on receiver demand. Robustness of the entire device may be enforced to help it beat all the environmental and climatic challenges at the war fronts and national borders. The rigorous analysis may be performed for integrating the proposed system with existing paraphernalia (used by army personnel) which may yield in budget-friendly and reliable implementation. Effective interfacing has been achieved and results suggest proper intimation of pulse rate and location as soon as pulse values drop below the pre-decided threshold or on receiver demand. Robustness of the entire device may be enforced to help it beat all the environmental and climatic challenges at the war fronts and national borders. The rigorous analysis may be performed for integrating the proposed system with existing paraphernalia (used by army personnel) which may yield in budget-friendly and reliable implementation $[1]$.

A Unique Health Care Monitoring System Using Sensors and Zig-Bee Technology can be established by looking at the paper that presents a remote measuring and monitoring system for electrocardiogram (ECG), lung functioning (spirometer), heart rate and temperature signals. The proposed system comprises the design and implementation with subsystems, viz. (A) The hardware module for acquisition, (B) The protocol for standard IEEE 802.15.4 ZigBee system, (C) The radio frequency (RF) trans-receiver circuits and finally (D) A displaying module (PC or mobile devices). Information is sent via IP (GPRS or Wi-Fi) to a database server containing clinical data, which can be accessed on the smartphone and can also be shared with the physician anytime to seek medical advice when needed. The goal of this project is to present the design of a compact sized and user-friendly smartphone accessory that can be accounted for in clinical care. Rapid economic and industrial development leads to an increased intensity in daily life, which brings people negative sentiments, such as nervousness, anxiety, and disturbance. These emotions along with changes of quickly lifestyle result that chronic cardiovascular diseases become the major adult illnesses. Recent studies have shown that using telemonitoring devices, together with patient communications, have a significant impact on the efficiency of care and quality of life, while decreasing re-hospitalization rates. Many medical centers nowadays install telemonitoring devices in patients' home to keep track of their medical condition. Although this is a major breakthrough, it still limits the patient from leaving home without being telemonitored to keep track of his medical condition. Smartphone technology breaks that limit as the smartphone when equipped with specific accessories and application can be more than just a phone but be also "Smart" to be a "telemonitoring device".

Almost all observation positions of human health (e.g., ECG, EEG, Blood, and Pressure) can be monitored by the related bio-microsystem device increasingly important to provide the high-quality healthcare monitoring.

Coming to the methodologies, firstly, we have Electrocardiogram (ECG), which is used to measure the rate and regularity of heartbeats, as well as the size and position of the chambers, the presence of any damage to the heart, and the effect of drugs and devices used to regulate the heart. Secondly, Heart Rate Estimation IC LM 358 is used for Heart Beat Sensor. Its dual low power operational amplifier Licit consists of a super bright red LED and the light detector. Thirdly, Temperature Sensor which is used to measure temperature, is an electronic device which provides a voltage analog of the temperature of the surface on which it is mounted. Lastly, Spirometer is the device that is used to make the measurements. Spirometry is a common office test used to diagnose asthma, chronic obstructive pulmonary disease (COPD) and certain other conditions that affect breathing. The advantage is it provides direction to choose appropriate node density, data transmission rate and communication duration for required performance while establishing a Zigbee sensor-based patient monitoring system in practice [2].

Real-Time Tracking and Health Monitoring of Soldiers using ZigBee Technology can be done by providing an ability to track the location and monitor the health of the soldiers in real time. It helps to minimize the time, search and rescue operation efforts of the army control unit. This system enables to army base station to track the location and monitor the health of soldiers using GPS module and wireless body area sensor networks (WBASNs), such as temperature sensor, heartbeat sensor, etc.. The data coming from sensors and GPS receiver is transmitted wirelessly using ZigBee module. Also, a soldier can ask for help from the control room and can communicate with other fellow soldier present within the wireless transmission and reception range. 
The system proposed by us is composed of two parts, one is a small portable unit for soldiers and other is for the army control unit. The soldiers unit consists of an ARM 7 (advanced RISC machine) microprocessor, GPS tracking device, ZigBee transceiver, heartbeat sensor, temperature sensor, etc.

A Zig-Bee module is a high-level communication device as well as a medium range communication devise used to create wireless personal area networks (WPANs) which is built from small and low power digital radios, and requires very low power to operate. ZigBee devices can transmit data over both medium and long range distances by passing data through a mesh network of intermediate ZigBee devices to reach up to more distant ones. As ZigBee networks are secured by a 128-bit symmetric encryption key, so the hacking of ZigBee modules is not possible for enemies and hackers as in GSM modules.

The GPS receiver is used to track the location of the soldiers through satellite. LM 35 which is a temperature sensor senses the body temperature of the soldiers. An IC LM358 will be used to sense the heartbeat of the soldiers. We will use LPC2148 microcontroller which is embedded and located with ARM 7 microprocessor to process all the received data through sensors and GPS receiver in real time. A ZigBee module will be used for wireless transmitting and receiving of data. The data received from GPS modem is an RS-232 level data, therefore, we have to use IC MAX232, which convert the RS-232 voltage level data to 5V TTL/CMOS level. An alphanumeric LCD display is used to display the data sensed from BSNs and coming from GPS modem.

The advantage of this paper is to provide a high level of safety to Soldiers life. This system will be suitable at all environmental conditions no matter what would be the weather condition. Continuous data preparations can provide the analysis for different soldiers. It is low cost, compact and less complex system which can be easily adopted by any military force. Due to the use of advanced technology and advanced equipment, this system will fulfill all the requirements of growing technologies [3].

GPS BASED SOLDIER TRACKING AND HEALTH INDICATION SYSTEM can be developed by using systems like biosensor networks that comprise various types of small, transmission modules, physiological sensors, and computational capabilities, and can thus facilitate low-cost wearable unobtrusive solutions for health monitoring systems. GPS (Global Positioning System) is used to determine the longitude and latitude nothing but the lines that are drawn on the map.

The devices such as weapons and firearms are being added to, and some militaries such as the Israeli Army which are exploring the possibility of embedding GPS devices so that squadron unit can actually track the real-time location in real time. Can be used for High-speed, short-range, soldier-to-soldier wireless communications can be used to implement that comes under RF module that will be required to record information on situational awareness, tactical instructions, and covert necessary related data during special operations reconnaissance and other missions.

This paper has an idea of tracking the soldier's location in real time and navigation between soldier to soldier such as knowing their speed, distance, height as well as health status of them during the war, which enables the army personnel to plan the war strategies. The base station gets the location of a soldier from GPS. It is necessary for the base station to guide the soldier on the correct path if he is lost on the battlefield. The control unit can take immediate action for the soldiers who are in trouble and send a message to the fellow soldier in order to rescue them. And hence can take immediate action by sending help for the soldier or sending backup for threat ahead. Using various biomedical sensor health parameters of soldier's are observed, the position and orientation of the soldier is trapped using the GPS module system [4].

Smart Soldier Assistance using WSN can be established by looking at the paper that presents the development of a system which will help wirelessly monitor the health parameters of a soldier and also send and receive messages wirelessly from the control unit. The System will use all the vital devices that can be used to sent and alert the message to the fellow soldier and rescue the injured one. The system comprises a biosensor signal conditioning module, a GPS module, and an LCD, a keypad. A Lab VIEW program has been developed to act as the central commanding station. This will process all the data from the individual soldiers, display the data and perform the functions mentioned. This will help in strategic planning of military missions and also reduce casualties. This paper also focuses on wireless health care monitoring and GPS position tracking for two soldiers. Also, some features for use in military applications have been added. The paper also talks about the implementation of a software solution using Lab VIEW for a complete soldier management system. Temperature Sensor, the surface body temperature is measured using TI's LM34 which is a high precision integrated circuit temperature sensor, whose output voltage varies linearly with Fahrenheit temperature. Its output is given to microcontroller's ADC. The GPS module was acquired from Rhydo Labs. It provides standard NMEA0183 strings. It provides current time, latitude, longitude, speed and travel direction that can 


\section{IJARCCE}

Vol. 8, Issue 2, February 2019

be recorded using accelerometer. However, only the latitude and longitude information was extracted from the strings. Keypad and 16X2 LCD A 4X4 matrix keypad and 16X2 LCD are used for providing an interface with the device. EZ430-RF2500 Target Board This module is used for broadcasting messages and information acquired by the Stellaris Launchpad. The Stellaris Launchpad sends information to this module via UART which is nothing but the A portable unit has been made which helps in continuously monitoring a healthy person's vitals, position and also facilitates communication within the network under stationary conditions [5].

\section{ARCHITECTURE DIAGRAM}

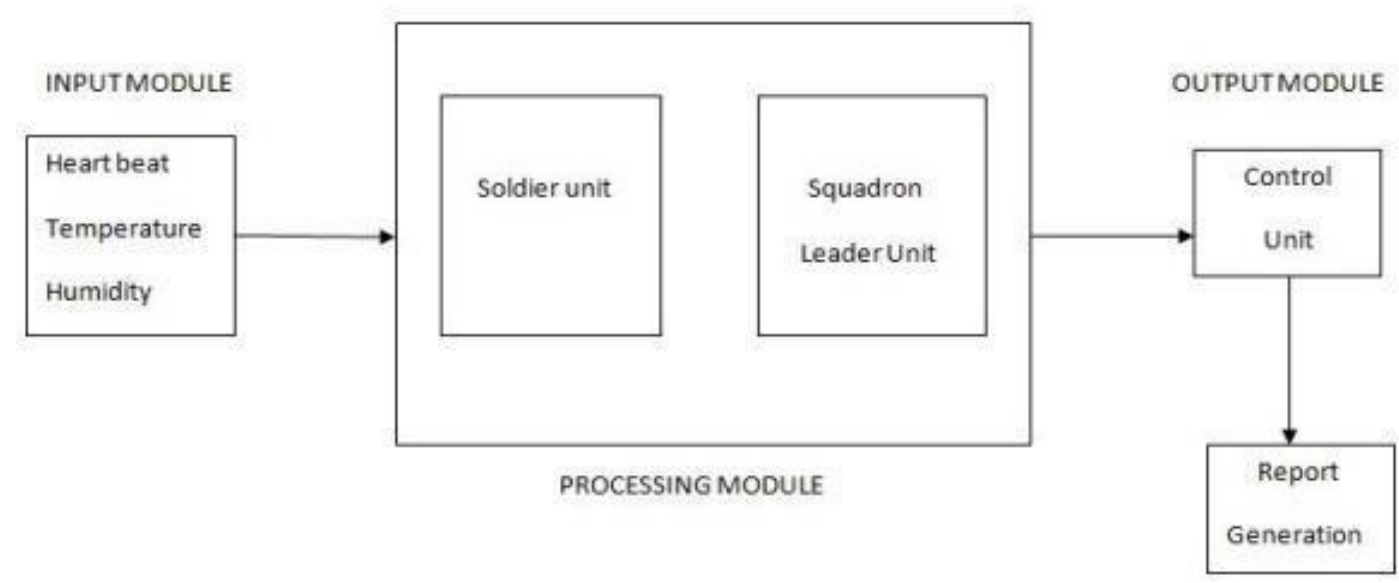

Figure 1- Architecture diagram

The figure shows the proposed system architecture diagram which consists of input module that contains various parameters such as temperature, humidity. a soldier's unit, a squadron leader unit, control unit and a report generation.

\section{CONCLUSION}

We hereby after surveying conclude that the above mentioned technologies would be used in our project description, when the soldier is in the battlefield he or she must wear the devise which in turn record all the health parameters of the soldier such the sensors attached to the devise will sense all the health parameters such as heartbeat, temperature and so on and will update it to the squadron, the squadron will in turn alert all the information gathered to the control unit and the control unit will compute the further analysis part using machine learning techniques and generate the report. The soldiers who are found to be in danger are rescued by sending the message to the squadron unit in order to track the location for those who become lost and get injured in the battlefield.

\section{REFERENCES}

[1] Hock Beng Lim, Di Ma, Bang Wang, Zbigniew Kalbarczyk, Ravishankar K. Iyer, Kenneth L. Watkin (2010) “A Soldier Health Monitoring System for Military Applications" International Conference on Body Sensor Networks, pp: (246-249)

[2] [2] Ekta Madhyan, Mahesh Kadam (2014) “A Unique Health Care Monitoring System Using Sensors and ZigBee Technology” International Journal of Advanced Research in Computer Science and Software Engineering, 4(6), pp: (501-509).

[3] Dineshkumar Jaiswar, Sanjna S. Repal (2015) "Real Time Tracking and Health Monitoring of Soldiers using ZigBee Technology: a Survey" International Journal of Innovative Research in Science, Engineering \& Technology, 4(7).

[4] Shruti Nikam, Supriya Patil, Prajkta Powar, V. S. Bendre (2013) “GPS Based Soldier Tracking and Health Indication System" International Journal of Advanced Research in Electrical, Electronics and Instrumentation Engineering, 2(3), pp: (1082-1088)

[5] M. Pranav Sailesh, C. Vimal Kumar, B. Cecil, B. M. Mangal Deep, P. Sivraj (2014) "Smart Soldier Assistance using WSN" International Conference on Embedded Systems - (ICES 2014), IEEE, pp: (244-249). 